\title{
Replacement of physiologically autoinhaled nitric oxide in intubated patients
}

\author{
L. Röhrig, R. Kuhlen, J. Baumert, R. Rossaint
}

Replacement of physiologically autoinhaled nitric oxide in intubated patients. L. Röhrig, R. Kuhlen, J. Baumert, R. Rossaint. C) ERS Journals Ltd 2003.

ABSTRACT: Autoinhaled nitric oxide (NO) is produced mainly in the upper airways. Orotracheal intubation disrupts the natural autoinhalation of NO from the naso- and oropharynx. The effect of disrupting and then replacing autoinhaled NO on arterial oxygenation was investigated in intubated patients.

Two groups of nine patients without lung disease were examined during anaesthesia using an inspired oxygen fraction of 0.50 . In both groups, the individually produced NO of the whole respiratory tract and the upper airways was determined. The amount of NO normally autoinhaled from the upper airways was replaced for $5 \mathrm{~min}$ after orotracheal intubation in one group.

The amount of NO from the upper respiratory tract was $47 \pm 19$ parts per billion (ppb) in the test group and the replaced NO concentration was $48 \pm 20 \mathrm{ppb}$. No significant increase in arterial oxygen tension could be detected during the replacement of the autoinhaled NO. Haemodynamic parameters remained unchanged. In the control group, the NO from the upper airways was $34 \pm 16 \mathrm{ppb}$. In contrast to the test group, it was not replaced after intubation.

These findings suggest that in healthy subjects the autoinhalation of nitric oxide does not play an important role in arterial oxygenation during anaesthesia.

Eur Respir J 2003; 21: 677-681.
Dept of Anaesthesiology, University Hospital, RWTH Aachen, Aachen, Germany.

Correspondence: L. Röhrig, Dept of Anaesthesiology, University Hospital, RWTH Aachen, Pauwelsstraße 30, 52074 Aachen, Germany. Fax: 492418860917

E-mail: LeenaRoehrig@web.de

Keywords: Arterial oxygenation autoinhalation inhalation

nitric oxide

respiratory physiology

ventilation/perfusion distribution

Received: July 292002

Accepted after revision: November 72002
Nitric oxide (NO) is formed within the respiratory tract by the enzyme NO synthase, which converts L-arginine to L-citrulline. NO has several physiological functions. It contributes to the modulation of the pulmonary vascular tone under physiological conditions and, perhaps more importantly, during hypoxia [1]. The major portion of endogenous NO is produced in the upper respiratory tract, particularly in the nasopharynx [2-4]. Under physiological conditions the NO produced in the oro- and nasopharynx is inhaled to some extent. Endotracheal intubation disrupts this autoinhalation from the upper airways. It is estimated that $\sim 50-100$ parts per billion (ppb) are autoinhaled during normal breathing $[2,5]$. In various diseases associated with pulmonary hypertension, inhalation of exogenous $\mathrm{NO}$ in supranormal concentrations is used as a therapeutic means to selectively decrease pulmonary vascular resistance in ventilated lung regions. Therefore, the ventilation/perfusion mismatch and the intrapulmonary right-to-left shunt are reduced, and consequently the arterial oxygenation is improved [6-10]. The physiological implication of autoinhaled NO in healthy humans is still unknown.

Bypassing the upper airway by orotracheal intubation disrupts the autoinhalation of NO from the oro- and nasopharynx. Therefore, the authors sought to investigate the physiological role of autoinhaled NO in patients in whom anaesthesia was induced and who were intubated. The hypothesis that the replacement of the exact amount of endogenous NO influences the arterial oxygenation was tested in a randomised, controlled study.

\section{Methods}

The study was approved by the hospital ethics committee and informed consent was obtained from each patient the day prior to the operation.

\section{Patients}

The patients investigated in this study were all undergoing a total hip endoprosthesis. Patients with an acute or a chronic lung disease, a severe cardiac disease or morbid obesity were excluded from this study. Smokers were also excluded.

\section{Technique of nitric oxide measurement and delivery}

For NO measurement the Exhalyzer system (ECO Physics $\mathrm{GmbH}$, Dürnten, Switzerland) was used. The Exhalyzer consists of the CLD 77 AM NO analyser and the spiroson scientific ultrasonic flowmeter. The CLD 77 AM works on the principle of chemiluminescence. It is capable of continuously measuring very small amounts of $\mathrm{NO}$ in real time with a low NO detection limit of $0.1 \mathrm{ppb}$. Therefore, NO is detected with a response time of $100 \mathrm{~ms}$ using a small sample flow of $350 \mathrm{~mL} \cdot \mathrm{min}^{-1}$. The accuracy of the measured flow and volume with the spiroson scientific flowmeter is not influenced by changes in gas composition, humidity or temperature.

To replace the inhaled NO, the Servo ventilator 300 NO-A (Siemens AG, Munich, Germany) was used. A built-in computerised system released NO during the inspiratory cycle from a tank filled with 20 parts per million (ppm) NO diluted in nitrogen (AGA Gas GmbH, Bottrop, Germany). Since the applied amounts of inhaled NO were very low, they were controlled with the CLD 77 AM [11]. The concentration was measured as close to the patient as possible to get the best estimate of the real amount of inhaled $\mathrm{NO}$, because significant fluctuations might be found along the inspiratory limb [12].

\section{Measurements}

When the patients entered the operating room, the routine monitoring was installed, including a three-lead electrocardiogram, 
noninvasive blood pressure measurement, a fingertip pulse oximeter and a capnographic device (Datex-Engstrom, Helsinki, Finland). All data was recorded and stored using a data acquisition system.

In addition to the noninvasive blood pressure measurement, a radial arterial catheter was inserted using local anaesthesia. Systolic, diastolic and mean arterial pressure (MAP) were measured with quartz transducers (pvb Medizintechnik, Kirchseeon, Germany) connected to the monitoring system (AS/3 Compact Monitor; Datex-Engstrom). The supine zero reference level was placed in mid-axilla position. Arterial blood samples were collected anaerobically and analysed immediately. The arterial oxygen tension $\left(\mathrm{Pa}_{2} \mathrm{O}_{2}\right)$ and arterial carbon dioxide tension as well as the $\mathrm{pH}$ were determined using standard blood gas electrodes (Rapidlab 860; Chiron diagnostics $\mathrm{GmbH}$, Tuttlingen, Germany).

Gas samples were taken from the inspiratory limb of the ventilator tubing and analysed (ABL505; Radiometer, Copenhagen, Denmark) at each measuring point. Thus, the fraction of inspired oxygen $\left(F \mathrm{I}, \mathrm{O}_{2}\right)$ could be determined exactly by means of measuring the partial pressure of oxygen in the inspired gas mixture and converting it to $F \mathrm{I}, \mathrm{O}_{2}$. The amount of $\mathrm{NO}$ in the ambient air and in the inspiratory gas mixture oxygen/air $\left(F \mathrm{I}, \mathrm{O}_{2}=0.50 \pm 0.01\right)$ was also measured. The detected concentrations were extremely low $(<1 \mathrm{ppb}$ in the ambient air and $<2 \mathrm{ppb}$ in the inspiratory gas mixture). According to the recommendations for NO measurement by the American Thoracic Society [13], NO concentrations $<5 \mathrm{ppb}$ in the inhaled air were referred to as NO-free. Nevertheless, the so-called zero-point calibration was performed with the help of the NO analyser. With this feature, the NO in the inspiratory gas mixture could be set to zero, so that subsequently, only the exhaled NO from the respiratory tract of the patients was measured after the zero-point calibration.

Using the computerised data acquisition system, data for exhaled NO and carbon dioxide as well as the flow values (minute volume and respiratory rate) were recorded and averaged during the 2 -min measuring period. The concentration of exhaled NO was determined at the end of expiration, analysing each single breath. Since the respiratory rate was 10 breaths $\cdot \mathrm{min}^{-1}, 20$ values were obtained to calculate the mean value. In order to achieve an adaptation to the ventilator setting, the measurements were started after a period of $5 \mathrm{~min}$.

In addition to the above mentioned parameters, the arterial blood samples, oxygen saturation and haemodynamic parameters (heart rate, blood pressure) were obtained for each period.

\section{Protocol}

As the autoinhaled doses of NO vary in different patients, the autoinhaled amounts of NO were determined individually. The autoinhaled NO was defined as the difference between the exhaled NO of the whole respiratory tract and the exhaled NO of the lower respiratory tract.

To detect the exhaled NO of the whole respiratory tract, a tight face mask covering the nose and mouth of the patient was used with an inspiratory gas mixture oxygen/air of 0.50 . The patients were asked to breathe normally. To guarantee homogenous conditions during the measurements, all patients were sedated and paralysed in the same way. They were sedated with continuous infusions of propofol $0.1 \mathrm{mg} \cdot \mathrm{kg}^{-1} \cdot \mathrm{min}^{-1}$ after a bolus of $2.0 \mathrm{mg} \cdot \mathrm{kg}^{-1}$ and remifentanil $0.1 \mu \mathrm{g} \cdot \mathrm{kg}^{-1} \cdot \mathrm{min}^{-1}$ and paralysed with vecuronium $0.1 \mathrm{mg} \cdot \mathrm{kg}^{-1}$. The ventilatory pattern remained unchanged throughout the whole protocol. Before and after intubation, an inspiratory gas mixture of oxygen/air of 0.50 , a tidal volume of $8 \mathrm{~mL} \cdot \mathrm{kg}^{-1}$ and a respiratory rate of $10 \mathrm{breaths} \cdot \mathrm{min}^{-1}$ were applied.

After the induction of anaesthesia, the patients were first ventilated with the tight face mask as described above. Orotracheal intubation was then performed to facilitate the isolated measurement of NO from the lower respiratory tract. After intubation, the ventilator Servo 300 NO-A was set to deliver the above mentioned ventilatory pattern. No positive end-expiratory pressure was applied. In the test group, the exact amount of individual autoinhaled NO was replaced for a period of $5 \mathrm{~min}$. In the control group, the amount of physiologically autoinhaled NO was also determined, but not replaced. The setting of the ventilator remained unchanged in both groups during the whole period. The effect on arterial oxygenation was registered at the end of the NO replacement. In the control group an arterial blood sample was also taken at this point in time. A flowchart of the protocol is presented in figure 1.

\section{Statistical analysis}

The data are presented as mean $\pm \mathrm{SD}$. Statistical comparisons were performed using Wilcoxon matched pairs signed rank test. A p-value of $<0.05$ was taken as statistically significant.

\section{Results}

Patient data and baseline values of the haemodynamic parameters are shown in table 1 . NO was measured in the ambient air as well as in the inspiratory gas mixture oxygen/ air $\left(F \mathrm{I}, \mathrm{O}_{2}=0.50 \pm 0.01\right)$. The $\mathrm{NO}$ detected in the ambient air was $0.6 \pm 0.3 \mathrm{ppb}$ and the detected NO in the inspiratory gas mixture was $1.7 \pm 0.8 \mathrm{ppb}$.

In the test group, the exhaled NO from the whole respiratory tract was $53 \pm 22 \mathrm{ppb}$, the exhaled NO measured after endotracheal intubation was $5 \pm 2 \mathrm{ppb}$ and the NO from the upper respiratory tract was $47 \pm 19 \mathrm{ppb}$. Accordingly, the amount of NO replaced in the test group was $48 \pm 20 \mathrm{ppb}$. In the control group, the exhaled NO determined from the whole respiratory tract was $38 \pm 16 \mathrm{ppb}$ and was $4 \pm 1 \mathrm{ppb}$ from the lower respiratory tract. Therefore, the NO from the upper

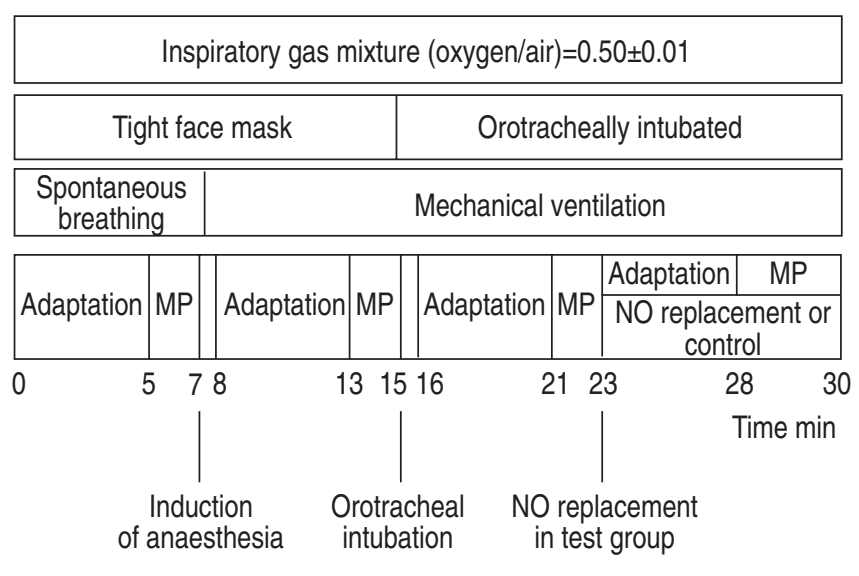

Fig. 1.-Flowchart of the protocol. During each measuring period (MP), the following parameters were determined: exhaled nitric oxide (NO), arterial oxygen tension, ventilation parameters (minute volume and respiratory rate) and haemodynamics (mean arterial pressure and heart rate). The flowchart illustrates the time sequence of the interventions and the periods of adaptation to the ventilator setting or to the NO replacement in the test group. 
Table 1.-Demographical data of the patients in the test group and the control group and baseline values of the haemodynamic parameters after induction of anaesthesia

\begin{tabular}{lcc}
\hline & Test group & Control group \\
\hline Age yrs & $71 \pm 10$ & $68 \pm 8$ \\
Height $\mathrm{m}$ & $1.66 \pm 0.06$ & $1.66 \pm 0.07$ \\
Weight $\mathrm{kg}$ & $70 \pm 11$ & $70 \pm 8$ \\
BMI kg $\cdot \mathrm{m}^{-2}$ & $25 \pm 3$ & $25 \pm 2$ \\
MAP mmHg & $88 \pm 13$ & $90 \pm 16$ \\
HR beats $\cdot \mathrm{min}^{-1}$ & $66 \pm 9$ & $63 \pm 11$ \\
\hline
\end{tabular}

Data are presented as mean \pm SD; BMI: body mass index; MAP: mean arterial pressure; HR: heart rate.

respiratory tract was determined to be $34 \pm 16 \mathrm{ppb}$ (table 2). The difference between the amounts of NO found in the upper airways of each group was not significant.

After the replacement of the NO from the upper airways in the test group no significant change in arterial oxygenation could be detected. At this point in time there was also no significant change in the arterial oxygenation in the control group (fig. 2 and table 3). Although the changes in arterial oxygenation between the individuals of each group were not significant, there was a significant difference in the $\mathrm{Pa}, \mathrm{O}_{2}$ values between the test group and the control group $(\mathrm{p}<0.05)$. Heart rate and MAP, as well as the ventilation parameters, remained virtually unchanged (table 3 ).

\section{Discussion}

In the present study, the authors investigated whether or not autoinhaled NO is involved in the modulation of pulmonary gas exchange in healthy humans. To examine the effect of autoinhaled NO, the subjects were deprived of the NO produced in the upper respiratory tract by means of endotracheal intubation. The results show that in healthy humans, the effect of autoinhaled NO seems to be of minor importance with respect to arterial oxygenation using an $F \mathrm{I}, \mathrm{O}_{2}$ of 0.50

Most of the endogenous NO is synthesised in the upper airways, particularly in the paranasal sinuses and the nose [2, 3]. Less than $10 \%$ of the NO is produced in the lower airways and the lungs [4]. Under physiological conditions, the NO produced in the upper airways reaches the lungs with inspiration and is thereby autoinhaled.

With the help of chemiluminescence, NO can be detected in the exhaled gases of several species [14]. In humans, the amount of NO in the exhaled air is reduced in smokers $[2,15]$ as well as in patients with cystic fibrosis and primary pulmonary dyskinesia $[3,16]$. The amount of NO in the exhaled air is greater in patients with asthma [3,5], instable chronic

Table 2. - Exhaled nitric oxide (NO) in the test group and the control group, as well as the amount of replaced NO in the test group

\begin{tabular}{lcc}
\hline & Test group & Control group \\
\hline NOUT+LT ppb & $52 \pm 21$ & $38 \pm 16$ \\
NOLT ppb & $5 \pm 2$ & $4 \pm 1$ \\
NOUT ppb & $47 \pm 19$ & $34 \pm 16$ \\
Replaced NO ppb & $48 \pm 20$ & Not replaced \\
\hline
\end{tabular}

Data are presented as mean \pm SD. NOUT+LT: NO from the whole respiratory tract; NOLT: NO from the lower respiratory tract; NOUT: NO from the upper respiratory tract; ppb: parts per billion. obstructive lung disease and bronchoectasis [16]. The measurement of exhaled NO with the technique of chemiluminescence has been found to be reproducible and differences were
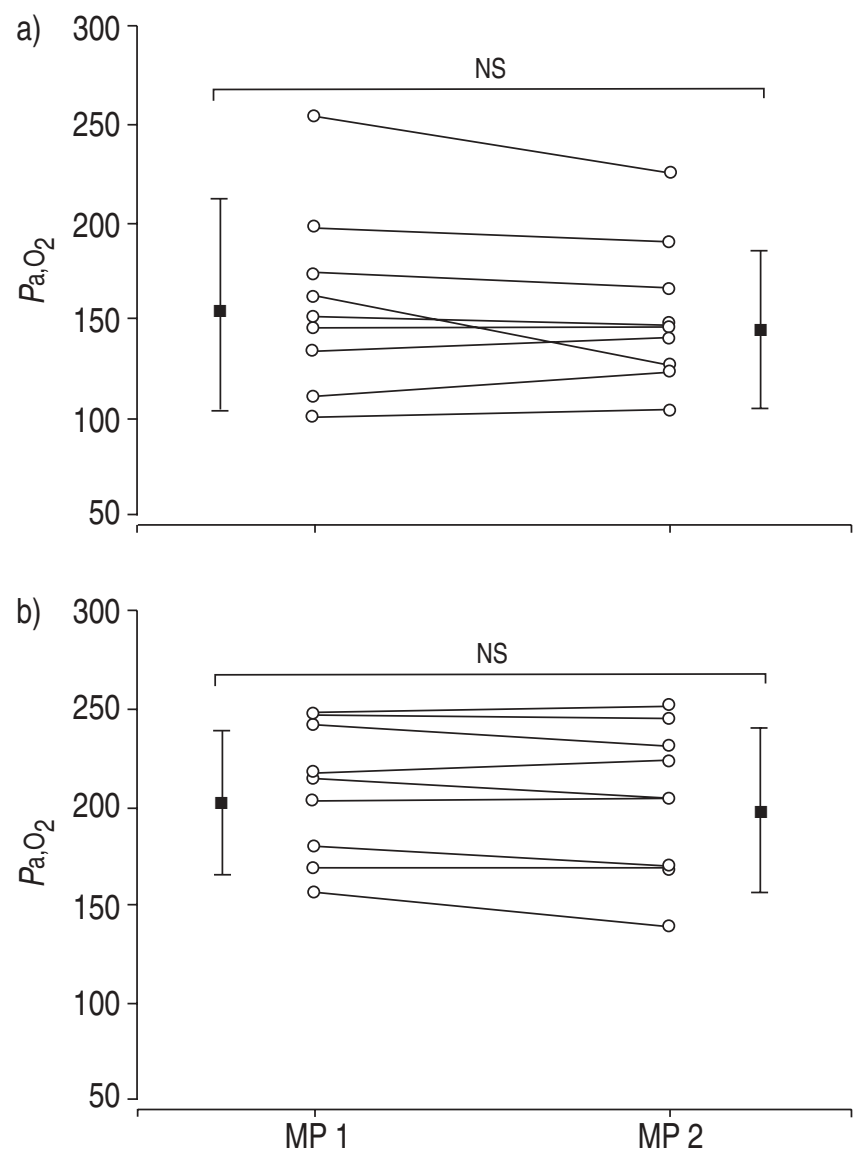

Fig. 2.-Individual changes of arterial oxygen tension $\left(\mathrm{Pa}, \mathrm{O}_{2}\right)$ in a) the test group and $b$ ) the control group at a constant inspiratory oxygen fraction of $0.50 \pm 0.01$ and an unchanged ventilator setting in the individual patient. The changes in the test group are nonsignificant (NS) after the replacement of the physiologically autoinhaled nitric oxide (NO; $\mathrm{p}=0.44)$. $\mathbf{\square}$ : mean value. Bars represent \pm SD. MP 1 : measuring period after endotracheal intubation; MP 2: measuring period after NO replacement in the test group for $5 \mathrm{~min}$.

Table 3. - Arterial oxygen tension $\left(\mathrm{Pa}, \mathrm{O}_{2}\right)$, haemodynamics and ventilation parameters in the test group and in the control group

\begin{tabular}{|c|c|c|c|c|}
\hline & \multicolumn{2}{|c|}{ Test group } & \multicolumn{2}{|c|}{ Control group } \\
\hline & MP 1 & MP 2 & MP 1 & MP 2 \\
\hline$P a, \mathrm{O}_{2} \mathrm{mmHg}$ & $158 \pm 44$ & $152 \pm 35$ & $208 \pm 31$ & $205 \pm 35$ \\
\hline$F \mathrm{I}, \mathrm{O}_{2} \mathrm{mmHg}$ & $0.50 \pm 0.01$ & $0.50 \pm 0.01$ & $0.50 \pm 0.01$ & $0.50 \pm 0.01$ \\
\hline$V^{\prime} \mathrm{E} \mathrm{L} \cdot \mathrm{min}^{-1}$ & $5.1 \pm 0.6$ & $5.1 \pm 0.6$ & $5.6 \pm 0.5$ & $5.6 \pm 0.5$ \\
\hline RR breaths $\cdot \min ^{-1}$ & $10 \pm 0$ & $10 \pm 0$ & $10 \pm 0$ & $10 \pm 0$ \\
\hline MAP $\mathrm{mmHg}$ & $85 \pm 12$ & $81 \pm 11$ & $86 \pm 14$ & $83 \pm 12$ \\
\hline HR beats $\cdot \mathrm{min}^{-1}$ & $63 \pm 8$ & $64 \pm 10$ & $62 \pm 12$ & $59 \pm 12$ \\
\hline
\end{tabular}

Data are presented as mean \pm SD. MP 1 : measuring period after endotracheal intubation; MP 2: measuring period after nitric oxide replacement in the test group for $5 \mathrm{~min} ; \mathrm{F}_{\mathrm{I}}, \mathrm{O}_{2}$ : inspired oxygen fraction; $V^{\prime} \mathrm{E}$ : minute volume; RR: respiratory rate; MAP: mean arterial pressure; HR: heart rate. The ventilator setting was not changed during the protocol in the individual patient. 
unrelated to sex or body mass index [17]. The amounts of exhaled NO in the present study are in accordance with previous studies $[4,18,19]$.

Several effects of NO have been investigated in recent years. The regulation of vasotonus is one of the most important functions of NO. Under conditions of increased pulmonary resistance, the value of supranormal doses of inhaled NO in the range of ppm has been shown in a number of studies [6-10, 20-22]. In patients with severe acute respiratory distress syndrome (ARDS), the inhalation of NO in supranormal concentrations has several effects. In cases of increased pulmonary vascular resistance, the inhalation of NO causes a selective pulmonary vasodilatation and, consequently, a redistribution of blood flow from shunt regions to well-ventilated lung regions. This leads to a reduction of the intrapulmonary shunt. The improvement of the ventilation/perfusion mismatch is revealed by an increase in arterial oxygenation [9]. These beneficial effects could also be observed during the inhalation of lower concentrations of NO in patients with severe ARDS. Even NO concentrations of $\sim 100 \mathrm{ppb}$, which are similar to physiologically autoinhaled amounts of NO in healthy humans, were capable of improving the ventilation/perfusion mismatch [2, 23, 24].

In contrast to conditions of pulmonary vascular dysfunction, supranormal $\mathrm{NO}$ in inspired air $\left(F \mathrm{I}, \mathrm{O}_{2}=0.21\right)$ seems to have no or almost no measurable effects on the normal pulmonary vasculature. The addition of exogenous NO to the inspired air did not influence the pulmonary vascular resistance in healthy dogs [25]. In awake sheep breathing normoxic air, the inhalation of NO did not reduce pulmonary vascular tone or enhance the usual reduction in pulmonary vascular resistance during exercise [26]. The influence of inhaled NO was also examined in healthy humans. During exercise under normoxic and hypoxic conditions no effect on gas exchange could be detected after inhalation of NO [27]. The absence of an effect of inhaled NO on healthy lungs breathing normoxic or even hypoxic air may reflect the presence of sufficient endogenous NO production.

As mentioned above, in lung diseases with regional alveolar hypoxia the replacement of low concentrations of NO similar to the physiologically autoinhaled NO clearly contributed to a redistribution of blood flow from nonventilated towards ventilated lung regions and improved the arterial oxygenation $[2,9,23,24]$.

In order to test the hypothesis of whether or not slightly lower amounts of autoinhaled NO would influence the arterial oxygenation in anaesthetised healthy humans, an experimental setting was used in which the autoinhalation of NO from the upper airways was prevented. The upper respiratory tract was bypassed by means of orotracheal intubation. The amount of NO exhaled from the upper airways, which is equivalent to the physiologically autoinhaled NO, was determined for each patient. Under these conditions, the replacement of the autoinhaled NO allowed the investigation of its physiological role. As shown in figure 2, no significant increase of $\mathrm{Pa}, \mathrm{O}_{2}$ could be determined after the replacement of the exact amount of autoinhaled NO in any patient in the test group. This result verifies that the autoinhaled NO does not play an important role in the regulation of the arterial oxygenation in healthy humans using an $F \mathrm{I}, \mathrm{O}_{2}$ of 0.50 .

The absence of an effect of the replaced autoinhaled NO may indicate that the NO production in the lower airways of healthy subjects is not impaired under physiological conditions. In contrast to patients with lung disease, the amount of NO present in the lungs seems to be sufficient to regulate the pulmonary gas exchange. Unfortunately, it cannot be guaranteed that the replaced amounts of NO completely reached the alveoli. Since significant fluctuations of inhaled NO can be found along the inspiratory limb [12], a further decline in the concentration of NO in the lower airways cannot be excluded.

It is well known that oxygen tension influences the pulmonary vascular tone. Hypoxia causes pulmonary vasoconstriction as a means of diverting the pulmonary blood flow away from regions of low oxygen tension and thereby optimising the ventilation/perfusion relationship [28]. Oxygen itself is a potent stimulus for pulmonary vasodilatation [29]. The applied $F \mathrm{I}, \mathrm{O}_{2}$ of 0.50 in this study led to supranormal $P a, O_{2}$ values. These $P \mathrm{a}, \mathrm{O}_{2}$ values are normal during anaesthesia, because general anaesthesia is commonly performed under hyperoxic conditions. Both oxygen and NO act as pulmonary vasodilators. Therefore, it cannot be excluded that the effect of NO replacement on arterial oxygenation was masked by a pre-existing pulmonary vasodilatation due to the high $F \mathrm{I}, \mathrm{O}_{2}$.

Since it was assumed that the NO-induced modulation of the pulmonary vascular tone is more important during hypoxia [1], the $F \mathrm{I}, \mathrm{O}_{2}$ of 0.50 in this study might have been too high to achieve a significant effect after the replacement of the autoinhaled NO. It must be stated, however, that NO replacement under conditions of a lower $F \mathrm{I}, \mathrm{O}_{2}$ would probably not have had a greater influence on the arterial oxygenation. Even under normoxic conditions, inhaled NO did not have an effect in healthy dogs or sheep [25, 26]. Since there might be differences among species, the effect of inhaled NO on healthy humans needs to be defined. In well-trained cyclists, inhaled NO had no effect on the gas exchange under normoxic as well as hypoxic conditions [27]. This result stresses that in healthy humans, even under hypoxic conditions, inhalation of NO does not play an important role in the pulmonary gas exchange.

Although the patients were randomised according to a welldefined randomisation protocol, significant differences were found between the $\mathrm{Pa}, \mathrm{O}_{2}$ values of the test group and the control group. All patients suffering from acute or chronic lung disease or severe heart disease and smokers were excluded from the study. No relevant differences concerning demographical data existed among the patients (table 1). Nevertheless, it cannot be assumed that other unknown influences might have been responsible for the differences between the $P \mathrm{a}, \mathrm{O}_{2}$ values of both groups. It was found that after the NO replacement in the test group, no significant increase of $\mathrm{Pa}, \mathrm{O}_{2}$ was seen in any of the patients. In addition, the $P \mathrm{a}, \mathrm{O}_{2}$ was not significantly different among the individuals of each group during any measuring period. This emphasises that both groups can be considered as homogenous. Therefore, the above-mentioned difference between the groups does not impair the informative value of this study.

Further studies are necessary to elucidate the biological implication of autoinhaled nitric oxide. New insights into the physiology and pathophysiology of autoinhaled nitric oxide may offer a better understanding and perhaps new indications for the therapeutic replacement of nitric oxide in the future.

\section{References}

1. Spriesterbach R, Grimminger F, Weissmann N, Walmrath D, Seeger W. On-line measurement of nitric oxide generation in buffer-perfused rabbit lungs. J Appl Physiol 1995; 78: 1502-1508.

2. Gerlach H, Rossaint R, Pappert D, Knorr M, Falke KJ. Autoinhalation of nitric oxide after endogenous synthesis in nasopharynx. Lancet 1994; 343: 518-519.

3. Lundberg JON. Airborne nitric oxide: Inflammatory marker and aerocrine messenger in man. Acta Physiol Scand 1996; 633: $1-27$.

4. Schedin U, Frostell C, Persson MG, Jakobsson J, Andersson G, Gustafsson LE. Contribution from upper and lower 
airways to exhaled endogenous nitric oxide in humans. Acta Anaesthesiol Scand 1995; 39: 327-332.

5. Kharitonov SA, Yates D, Robbins RA, Logan Sinclair R, Shinebourne EA, Barnes PJ. Increased nitric oxide in exhaled air of asthmatic patients. Lancet 1994; 343: 133-135.

6. Journois D, Pouard P, Mauriat P, Malhere T, Vouhe P, Safran D. Inhaled nitric oxide as a therapy for pulmonary hypertension after operations for congenital heart defects J Thorac Cardiovasc Surg 1994; 107: 1129-1135.

7. Kinsella JP, Neish SR, Shaffer E, Abman SH. Low-dose inhalational nitric oxide in persistent pulmonary hypertension of the newborn. Lancet 1992; 340: 819-820.

8. Pepke-Zaba J, Higenbottam TW, Dinh-Xuan AT, Stone D, Wallwork J. Inhaled nitric oxide as a cause of selective pulmonary vasodilatation in pulmonary hypertension. Lancet 1991; 338: 1173-1174.

9. Rossaint R, Falke KJ, Lopez F, Slama K, Pison U, Zapol WM. Inhaled nitric oxide in adult respiratory distress syndrome. N Engl J Med 1993; 322: 399-405.

10. Rossaint R, Gerlach H, Schmidt-Ruhnke H, et al. Efficacy of nitric oxide inhalation in severe ARDS. Chest 1995; 107: 1107-1115.

11. Kuhlen R, Busch T, Kaisers U, et al. Evaluation of two prototypes for inhalation of nitric oxide during mechanical ventilation with different tidal volumes. Clin Intensive Care 1997; 8: 171-177.

12. Kuhlen R, Busch T, Max M, Reyle-Hahn M, Falke K, Rossaint R. Fluctuations of inspired concentrations of nitric oxide and nitrogen dioxide during mechanical ventilation. Crit Care 1999; 3: 1-6.

13. American Thoracic Society. Recommendation for standardized procedures for the online and offline measurement of exhaled lower respiratory nitric oxide and nasal nitric oxide in adults and children - 1999. Am J Respir Crit Care Med 1999; 160: 2104-2117.

14. Gustafsson LE, Leone AM, Persson MG, Wiklund NP, Moncada S. Endogenous nitric oxide is present in the exhaled air of rabbits, guinea pigs and humans. Biochem Biophys Res Comm 1991; 181: 852-857.

15. Busch T, Kuhlen R, Knorr M, et al. Nasal, pulmonary and autoinhaled nitric oxide at rest and during moderate exercise. Intensive Care Med 2000; 26: 391-399.

16. Kharitonov SA, Barnes PJ. Clinical aspects of exhaled nitric oxide. Eur Respir J 2000; 16: 781-792.

17. Sanchez Gutierrez C, Romero Romero B, Jimenez Solis R, Castillo Gomez J. Reproducibility of a technique for measuring nitric oxide in exhaled air in healthy subjects. Arch Bronconeumol 2001; 37: 371-374.

18. Brett SJ, Evans TW. Measurement of endogenous nitric oxide in the lungs of patients with the acute respiratory distress syndrome. Am J Respir Crit Care Med 1998; 157: 993-997.

19. Hogman M, Drca N, Ehrstedt C, Merilainen P. Exhaled nitric oxide partitioned into alveolar, lower airways and nasal contributions. Respir Med 2000; 94: 985-991.

20. Bigatello LM, Hurford WE, Kacmarek RM, Roberts JD Jr, Zapol WM. Prolonged inhalation of low concentrations of nitric oxide in patients with severe adult respiratory distress syndrome. Effects on pulmonary hemodynamics and oxygenation. Anesthesiology 1994; 80: 761-770.

21. Frostell C, Fratacci MD, Wain JC, Jones R, Zapol WM. Inhaled nitric oxide. A selective pulmonary vasodilator reversing hypoxic pulmonary vasoconstriction. Circulation 1991; 83: 2038-2047.

22. Pison U, Lopez FA, Heidelmeyer CF, Rossaint R, Falke K Inhaled nitric oxide selectively reverses hypoxic pulmonary vasoconstriction without impairing pulmonary gas exchange. J Appl Physiol 1993; 74: 1287-1292.

23. Gerlach H, Rossaint R, Pappert D, Falke KJ. Time-course and dose-response of nitric oxide inhalation for systemic oxygenation and pulmonary hypertension in patients with adult respiratory distress syndrome. Eur J Clin Invest 1993 23: 499-502.

24. Gerlach H, Pappert D, Lewandowski K, Rossaint R, Falke KJ. Long-term inhalation with evaluated low doses of nitric oxide for selective improvement of oxygenation in patients with adult respiratory distress syndrome. Intensive Care Med 1993; 19: 443-449.

25. van Camp JR, Yian C, Lupinetti FM. Regulation of pulmonary vascular tone by endogenous and exogenous nitric oxide. Ann Thorac Surg 1994; 58: 1025-1030.

26. Koizumi T, Gupta R, Banerjee M, Newman JH. Changes in pulmonary vascular tone during exercise. Effects of nitric oxide (NO) synthase inhibition, L-arginine infusion and NOinhalation. J Clin Invest 1994; 94: 2275-2282.

27. Sheel AW, Edwards MR, Hunte GS, McKenzie DC. Influence of inhaled nitric oxide on gas exchange during normoxic and hypoxic exercise in highly trained cyclists. J Appl Physiol 2001; 90: 926-932.

28. Marshall BE. Pulmonary vasoconstriction. Acta Anaesthesiol Scand 1990; 34: 37-41

29. Gosche JR. Oxygen dilation in fetal pulmonary arterioles: role of $\mathrm{K}(+)$ channels. J Surg Res 2001; 97: 159-163. 